\title{
Efficacy of relaxation techniques in the reduction of tension, anxiety and stress perceived by patients with cancer under chemotherapy treatment
}

\author{
Flor Esmeralda Larios-Jiménezi,2, Leivy Patricia González-Ramírez ${ }^{3}$, Roberto Montes-Delgado4, \\ Fabiola González-Betanzos ${ }^{5}$, Andrés de Jesús Morán-Mendoza ${ }^{6}$, Pedro Solano-Murillo, \\ Antonio Oceguera-Villanueva ${ }^{8}$, Mónica Montero-Ramírez ${ }^{8}$ and Adrián Daneri-Navarro ${ }^{9 *}$
}

${ }^{1}$ Alumna del Doctorado Interinstitucional en Psicología, Campus Colima, Universidad de Colima, Colima; ${ }^{2}$ Departamento de Disciplinas Filosóficas, Metodológicas e Instrumentales, Centro Universitario de Ciencias de la Salud, Universidad de Guadalajara, Guadalajara; ${ }^{3}$ Centro Universitario de Tonalá, Universidad de Guadalajara, Tonalá; ${ }^{4}$ Facultad de Psicología, Universidad de Colima, Colima; ${ }^{5}$ Facultad de Psicología, Universidad Michoacana de San Nicolás de Hidalgo, Morelia; ${ }^{6}$ UMAE Hospital de Ginecoobstetricia; ${ }^{7}$ UMAE Hospital de Especialidades, CMNO, IMSS; ${ }^{8}$ Instituto Jalisciense de Cancerología; ${ }^{9}$ Departamento de Fisiología, Centro Universitario de Ciencias de la Salud, Universidad de Guadalajara, Guadalajara. México

\begin{abstract}
Introduction: Cancer constitutes a public health problem worldwide. Attention to patients with cancer includes treatments such as chemotherapy, which may generate emotional negative reactions. Relaxation techniques have shown to reduce anxiety and stress in this population. Materials and Methods: 552 patients of the chemotherapy areas of three hospitals of Metropolitan Guadalajara zone were trained in diaphragmatic breathing and guided imagery. Patients were assessed with an visual analogue scale, which scored their tension, anxiety or perceived stress before and after relaxation training. Results: The mean obtained by the visual analogue scale was 3.24 (SD: 2.8) before and 1.2 (SD: 1.9) after relaxation training respectively $(p<0.001)$. Moreover, differences in means among patients in different hospitals $(p<0.001)$ were observed. Conclusions: Our results highlight the effectiveness of relaxation techniques implemented in patients receiving chemotherapy, reducing perceived stress, anxiety and tension. Health professionals can train patients on the use of relaxation techniques during their hospital visits. The generalized practice of these techniques by the patients will improve their state of wellbeing, by reducing the emotional affections caused by cancer diagnosis and the treatments.
\end{abstract}

Key words: Diaphragmatic breathing. Guided imagery. Cancer. Chemotherapy. Patient navigation program.

\section{Correspondence:}

${ }^{*}$ A. Daneri-Navarro

E-mail: daneri@cucs.udg.mx
Available online: $30-05-2019$ Gac Mex Oncol. 2018;17:42-47 www.gamo-smeo.com 1665-9201/@ 2018 Sociedad Mexicana de Oncología. Published by Permanyer México. This is an Open Access article under the terms of the CC BY-NC-ND license (http://creativecommons.org/licenses/by-nc-nd/4.0/). 


\section{Introduction}

Cancer is a health problem with high mortality in developing countries owing to late diagnosis ${ }^{1}$. One of the most widely used treatments to treat advanced stages of the disease is chemotherapy. When faced with chemotherapy, cancer patients may experience stress, anxiety, fear, apprehension, anger and frustration, among other emotional reactions, deriving from a feeling of lack of control of the situation and an accentuated vision of cancer as a disease that is synonymous with death ${ }^{2,3}$. Emotional reactions can hinder the process of adaptation to treatment ${ }^{4,5}$, and without appropriate and timely treatment, patients can end up in situations of depression, anxiety, sleep problems, poor therapeutic adherence and quality of life deterioration ${ }^{6,7}$.

Psychological stress is the result of a relationship between the individual and an environment that is evaluated as threatening or overwhelming. It is a perception by the individual that the environment demands too much of him/her, exceeds his/her resources and is detrimental to his/her well-being. It should be bore mind that individual differences play a significant role in stress processes. Predisposing characteristics, motivations, attitudes and experiences determine how people perceive and evaluate demands. This evaluation influences on coping styles, emotional responses and, in the long term, on health outcomes ${ }^{8}$.

In recent decades, relaxation techniques have been used with great success and as a complement to pharmacological treatments ${ }^{9,10}$. Particularly, in patients on chemotherapy treatment, these techniques have been considered an important resource, even for symptoms such as nausea and vomiting ${ }^{11}$. Learning these techniques is simple; however, training and supervision by the health professional is initially required through brief interventions. Guided imagery and diaphragmatic breathing are widely used to reduce comorbidities such as stress, anxiety and depression, and for this reason they were selected for the present study ${ }^{12,13}$. In addition, they are useful for: a) relieving muscle tension, pain and discomfort, b) reducing sympathetic activation, c) achieving a state of serenity and eliminating subjective tension, d) being able to sleep and rest, e) focusing on the bodily sensations of here and now and diverting attention from catastrophic thoughts, and g) as a coping strategy to give a sense of control of situations ${ }^{14}$.

Relaxation through diaphragmatic breathing is easy to learn and, once trained, it can be practiced in any context. It is a skill that requires twice or thrice daily practice for approximately 15 or 20 minutes to achieve the desired results. It involves carrying out five basic steps: a) getting the inspired air to go to the lower part of the lungs, b) getting the inspired air to go to the lower and middle part of the lungs, c) achieving a full inspiration, d) obtaining a more complete and regular expiration, and e) establishing adequate respiratory alternation ${ }^{14}$. On the other hand, guided imagery consists in the conscious and voluntary creation of mental representations of objects, places, people, situations or oneself.

The purpose of the present study was to assess the efficacy of the relaxation techniques used to reduce the symptoms of tension, anxiety or stress perceived by cancer patients who received chemotherapy on an outpatient basis at three hospitals from Guadalajara, Jalisco, Mexico.

\section{Material and methods}

\section{Participants and scenarios}

Between April 2014 and November 2015, diaphragmatic breathing and guided imagery techniques were applied to 588 patients in the chemotherapy areas of three hospitals in the Metropolitan Area of Guadalajara: Specialty Hospital and Obstetrics and Gynecology Hospital of the Mexican Institute of Social Security, which serves affiliated population, as well as in the Jalisco Institute of Cancerology, whose patient population has no Social Security. At the Specialty Hospital chemotherapy area, drug administration services are offered to cancer patients, as well as to patients with rheumatoid and immune system diseases; treatment administration is carried out in cubicles for a maximum of three people per area. In the other two hospitals, the chemotherapy units offer treatments only to population with cancer, and in these institutions, the rooms are large and are shared by several patients, with a group environment being perceived. For the present work, 36 evaluations were excluded because they involved patients with Lupus or patients who did not complete the evaluation scale at any of the assessment time-points.

\section{Procedure}

For the application of the relaxation techniques, nine psychology, nursing and medicine students who provided social service or professional practices within the navigation program to help cancer patients and their families at the University of Guadalajara were trained to apply the program and supervised. This program, 
hereinafter referred to as "patient navigation program", has as its central objective to benefit the patient with cancer and his/her family by facilitating, with professionalism and warmth, the overcoming of barriers in order to achieve comprehensive and timely care and, through interinstitutional and social links, have access to services such as psychoeducation, emotional accompaniment and training in relaxation techniques. In addition, the program has the purpose to contribute to the training of human health resources, to promote ethical treatment and to improve healthcare practice through research.

Prior to the application of the relaxation techniques, a climate of trust (rapport) was established with the patients who were receiving their chemotherapy treatment. Then, they were explained the benefits of practicing relaxation techniques within the oncological process and were invited to participate in relaxation and complete the first part of the assessment to participate in the present study.

During the intervention, using the modeling technique, the facilitator instructed the participants to adequately perform diaphragmatic breathing. Once they performed it correctly, they were invited to close their eyes and attentively listen to the facilitator's voice, who guided them through a particular scenario, which was chosen according to the characteristics of the group or preferences of the people with whom the exercise was being carried out. Subsequently, the characteristics of the chosen scenario (forest, beach, tulip field, tropical forest or swimming with dolphins) were described by the facilitator, modulating his/her voice, alternating short and long pauses according to the characteristics of the scenario in question. Finally, a subsequent evaluation was carried out and feedback on patients' experience was encouraged. The entire procedure lasted between 40 and 60 minutes.

\section{Instrument}

A visual analogue scale was designed to measure the level of tension, anxiety or stress the participant was feeling before and after the relaxation technique application. The instrument was planned to be simple and quick to answer. The first part asks for sociodemographic and clinical information such as age, place of residence, level of education, marital status, diagnosis, chemotherapy cycle or number that is being received, and it also inquires whether it is the first time the instrument is being applied or how many times has been it applied before by any navigator. In the "Before the technique" instructions, the patient is asked to rate his/ her level of tension, anxiety or stress at the moment in a scale of 0 to 10, where 0 is very relaxed and does not feel any tension, anxiety or stress, and 10 represents the most intense sensation the patient has had in his/her life with regard to the indicated conditions. After the relaxation technique is applied, the patients are asked to read the "after the technique" instructions and re-score their level of tension, anxiety or stress under the same criteria, with the possibility of finally writing down in a narrative way some observation or recommendation regarding the applied technique.

\section{Statistical analysis}

The analyses were carried out using the Statistical Package for Social Sciences (SPSS ${ }^{\circledR}$ ) program, version 20.0. Frequencies, percentages, means and SD were obtained to describe patient sociodemographic and clinical characteristics.

The Kolmogorov-Smirnov and Shapiro-Wilk normality tests were used, with normal distribution of data being found. Student's t test was used for paired data to compare the means of the scores obtained in the relaxation technique pre- and post-application assessment with the visual analogue scale.

A variable was created based on the differences obtained between the assessments before and after the relaxation application. With this variable, a t-test for independent samples was carried out to find out if there were differences in tension, anxiety or stress reduction between patients by gender and between those "without Social Security" and "with Social Security".

\section{Ethical aspects}

There is a collaboration agreement between the University of Guadalajara and the participating hospitals to offer various services to cancer patients through the patient navigation program. In accordance with article 11.3 of the Official Mexican Standard NOM-012SSA3-2012 ${ }^{15}$, which establishes the criteria for the execution of research projects for human health in cases of investigations without risk or with minimum risk, informed consent is not required. The purpose of publishing the evaluation results was verbally explained to the patients, clarifying that the information would be confidential and explaining that they were free withdraw from the study at any time. In addition, the research was conducted in adherence to the ethical principles established by 
Table 1. Sample characteristics central tendency, dispersion and frequency measures and percentages

\begin{tabular}{|c|c|c|}
\hline & $\mathrm{m} \pm S D^{*}$ & n $(\%)^{* *}$ \\
\hline Age & $50 \pm 15$ & \\
\hline $\begin{array}{l}\text { Gender } \\
\text { Females } \\
\text { Males }\end{array}$ & & $\begin{array}{c}459(83.2) \\
93(16.8)\end{array}$ \\
\hline $\begin{array}{l}\text { Social Security } \\
\text { Without Social Security } \\
\text { With Social Security }\end{array}$ & & $\begin{array}{l}284(51.4) \\
247(44.8)\end{array}$ \\
\hline $\begin{array}{l}\text { Marital status } \\
\text { Single } \\
\text { Married } \\
\text { Divorced } \\
\text { Widowed } \\
\text { Cohabitating }\end{array}$ & & $\begin{array}{c}122(22.1) \\
348(63) \\
25(4.5) \\
46(8.3) \\
4(0.7)\end{array}$ \\
\hline $\begin{array}{l}\text { Occupation } \\
\text { Employed } \\
\text { Unemployed }\end{array}$ & & $\begin{array}{l}177(32.1) \\
339(61.4)\end{array}$ \\
\hline
\end{tabular}

the Declaration of Helsinki for research with human beings.

\section{Results}

Total sample included 552 cancer patients. Average age of participants was $50 \pm 15$ years. Around $83 \%$ of the sample were women and $51 \%$ did not have Social Security (Table 1). Visual analogue scale mean scores were 3.24 (SD: 2.8) in the pre-relaxation assessment and 1.2 (SD: 1.9) in the post-relaxation assessment (Fig. 1). A statistically significant difference was obtained between the means of these two evaluations, with a $p$-value $<0.001$.

It should be noted that, of the 552 assessed patients, $389(70.5 \%)$ perceived a lower level of tension, anxiety and stress, $149(27 \%)$ had the same score before and after the technique and only $14(2.5 \%)$ scored higher in the perception of tension, anxiety or stress after the relaxation technique.

With the variable generated with the difference between the assessment before and after the relaxation technique in the visual analogue scale, no statistically significant differences were obtained according to gen$\operatorname{der}(p=0.11)$. Conversely, a statistically significant difference was found between patients "without Social Security" and "with Social Security", with a p-value $<0.001$, with an average of $3.15 \pm 3.04$ in the previous

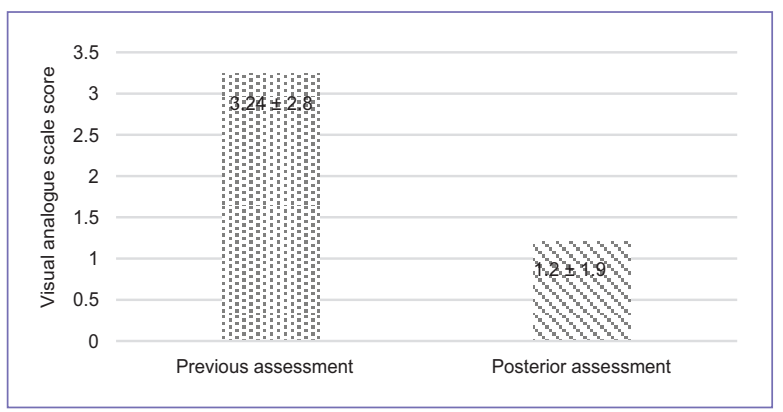

Figure 1. Comparison of scores before and after the use of the relaxation technique.

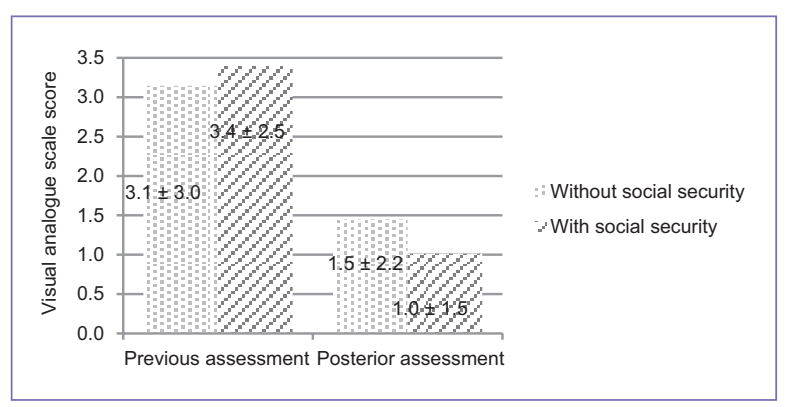

Figure 2. Comparison of the levels of tension, anxiety or stress perceived between people without and with Social Security.

evaluation and $1.45 \pm 2.17$ in the subsequent evaluation being observed for the people "without Social Security", whereas in the people "with Social Security", average values were $3.4 \pm 2.46$ and $1.01 \pm 1.51$, respectively (Fig. 2).

\section{Discussion}

Our results show a decrease in the levels of tension, anxiety or stress perceived by cancer patients after the application of relaxation techniques during chemotherapy. These patients usually experience, among other physical and psychological symptoms, tension, anxiety or stress during treatment administration ${ }^{16-18}$, and stress in particular has been associated with a deterioration of the immune system capacity to defend the body ${ }^{19,20}$. An experimental study that assessed the benefits of guided imagery daily practice for a week in patients with breast cancer, showed that those who received the relaxation training showed a significant decrease in insomnia, pain, anxiety and depression, in contrast with those who did not receive such training, who had more symptoms of nausea, vomiting and loss of appetite, 
among others ${ }^{16}$. Similar results were obtained in the study by Masafi, Rezaeib and Ahadic ${ }^{21}$, who observed a decrease in the level of anxiety in the experimental group and an increase in the control group. In addition to relaxation training, Masafi et al. associated the use of biofeedback in the experimental group of breast cancer patients after measurement in three chemotherapy sessions.

On the other hand, Charalambous et al. ${ }^{9}$ carried out a longitudinal study to verify the efficacy of guided imagery and progressive muscular relaxation for the treatment of anxiety and depression in patients with breast and prostate cancer who received chemotherapy. They accompanied their analyses with the use of biomarkers such as cortisol and the amylase- $\alpha$ enzyme, with the combination of techniques for mediating the physiological response and the reduction of perceived stress being found effective. These authors also highlight the scientific evidence on the high incidence of anxiety and depression associated with patients receiving chemotherapy, which threatens treatment compliance and thereby quality of life. They propose that the intervention of these patients demands a more comprehensive treatment, beyond the sole use of pharmacological treatment.

Relaxation techniques have also been tested as a resource for the treatment of pain in cancer patients. One study used the guided imagery technique for the treatment of pain in patients with cancer, with the technique being found to be effective in two thirds of their sample. In addition, the patients of this study reported the added benefit of a decrease in pain-associated anxiety, since the technique favored the sense of control over this symptom, contributed to a change in the perception of pain and to increase treatment efficacy ${ }^{10}$.

Regarding diaphragmatic breathing, Hayama et al. ${ }^{22}$ carried out a before/after evaluation study using only diaphragmatic relaxation training in women diagnosed with any type of gynecological cancer who received adjuvant chemotherapy. Twenty-five patients comprised their sample, which was divided in two groups. One of the groups received diaphragmatic breathing training. The anxiety-stress and fatigue scores in patients of the experimental group were significantly lower in the posttest assessment than in the control group. On the other hand, Qing-Hua Song et al. ${ }^{23}$ used diaphragmatic breathing combined with progressive muscle relaxation and reported an experimental study that showed that training in these techniques reduces adverse psychological reactions such as anxiety.

Of note, in the aforementioned studies, it is the Eastern countries that promote the use of relaxation techniques to a larger extent. Our study contributes to raise awareness in the West on the benefits that training in these techniques has on cancer patients receiving chemotherapy.

Regarding the statistically significant differences in patients with Social Security in comparison with those who do not have it, it is important to note that, in the first scenario, some patients receive treatment in individual rooms or in a context of up to three people, which promotes a climate of greater privacy. Isolation during the application of relaxation techniques favors an environment of tranquility and can contribute to reduce distractors that alter concentration. Unfortunately, due to the high demand for care in tertiary care public hospitals of our country, sometimes privacy during treatment administration is not viable.

The present study has some limitations. First, the lack of a control group, which prevents the differences in the scores of the pretest and posttest evaluation from being contrasted. Second, it does not report the follow-up of patients who received the diaphragmatic breathing and guided imagery techniques. Currently, as part of a project to evaluate the efficacy of the navigation program actions, work is being done on this aspect: on one hand, to find out whether the recommendation to practice these techniques at home or other environments is followed by patients and, on the other, to verify the long-term effect of the use of these relaxation techniques. Finally, the existence of multiple health professionals applying the technique might be a confounding variable; therefore, we consider it would be worthwhile carrying out a more rigorous and controlled follow-up, to avoid, for example, that those who apply the technique also cause differences between hospitals.

\section{Conclusion}

Our study adds to the scientific evidence for promoting the training of health personnel on the application of these techniques, with the purpose to improve medical attention and comprehensive care for cancer patients receiving chemotherapy treatment. In addition, it demonstrates the efficacy of relaxation techniques to reduce tension, anxiety or stress perceived during chemotherapy administration. Experimental studies have already proven the efficacy of relaxation in the treatment of pain, nausea, vomiting, insomnia and other symptoms in similar samples. The differences in the decrease of tension, anxiety or stress between the different hospitals of our study suggest the influence of 
the context to facilitate the application of the techniques, with more private environments that reduce the probability of distractions or interruptions during practice being preferable.

Relaxation techniques are strategies that can be trained and administered by interested health personnel, with the advantages of not being costly or time-consuming, in addition to contributing to well-being in this and other hospital care contexts, and may increase in the user the perception of quality care by the health team.

\section{Conflict of interests}

The authors declare not having any conflict of interests.

\section{Acknowledgements}

To "Cruzada AVON contra el cancer de mama" for the funding provided for the care of women with breast cancer and their families, who are looked after through the patient navigation program.

To Susan G. Komen for the CURE ${ }^{\circledR}$ (Grant Number SPMEX1201), for the financial support for the design and implementation of the patient navigation program in Guadalajara, Mexico.

To the Latin American Cancer Research Network and to the National Cancer Institute of the United States, as well as to the National Institutes of Health.

To the Consejo Nacional de Ciencia y Tecnología (CONACYT) through the Interinstitutional Doctorate in Psychology at Universidad Autónoma de Aguascalientes, Universidad de Colima, Universidad de Guadalajara, Universidad de Guanajuato and Universidad Michoacana de San Nicolás de Hidalgo.

As well as to navigators Sara Karen Rivas Medina, Lizeth Castellón Guerrero, Carina García Martínez, Alan Tiscareño Rodríguez, Daniel Díaz López, Oscar Daniel Valdez Pulido, Ruth Paulina Aguilar Rivera, Jacqueline Ramírez, Omar Víctor Ernesto Rubio Plascencia and Alma Guillermina Maya González.

\section{References}

1. Torre LA, Bray F, Siegel RL, Ferlay J, Lortet-Tieulent J, Jemal A. Global cancer statistics, 2012. CA Cancer J Clin. 2015;65(2):87-108.
2. Whitaker KL, Cromme S, Winstanley K, Renzi C, Wardle J. Emotional responses to the experience of cancer 'alarm' symptoms. Psychooncology. 2016;25(5):567-73.

3. Ashing-Giwa KT, Padilla G, Tejero J, Kraemer J, Wright K, Coscarelli A et al. Understanding the breast cancer experience of women: a qualitative study of African American, Asian American, Latina and Caucasian cancer survivors. Psychooncology. 2004;13(6):408-28.

4. Grassi L, Berardi MA, Ruffilli F, Meggiolaro E, Andritsch E, Sirgo A, et al. Role of psychosocial variables on chemotherapy-induced nausea and vomiting and health-related quality of life among cancer patients: a European study. Psychother Psychosom. 2015;84(6):339-47.

5. Meraner V, Gamper EM, Grahmann A, Giesinger JM, Wiesbauer P, Sztankay M, et al. Monitoring physical and psychosocial symptom trajectories in ovarian cancer patients receiving chemotherapy. BMC Cancer. 2012;12:77.

6. Ates O, Soylu C, Babacan T, Sarici F, Kertmen N, Allen D, et al. Assessment of psychosocial factors and distress in women having adjuvant endocrine therapy for breast cancer: the relationship among emotional distress and patient and treatment-related factors. Springerplus. 2016;5:486.

7. Miles A, McClements PL, Steele RJ, Redeker C, Sevdalis N, Wardle J. The psychological impact of a colorectal cancer diagnosis following a negative fecal occult blood test result. Cancer Epidemiol Biomarkers Prev. 2015;24(7):1032-8.

8. Lazarus SR, Kanner AD, Folkman S. Emotions: A cognitive-phenomenological analysis En: Plutchik R, Kellerman H, editores. Theories of Emotion. EE.UU.: Elsevier; 1980.

9. Charalambous A, Giannakopoulou M, Bozas E, Paikousis L. A randomized controlled trial for the effectiveness of progressive muscle relaxation and guided imagery as anxiety reducing interventions in breast and prostate cancer patients undergoing chemotherapy. Evid Based Complement Alternat Med. 2015;2015:270876.

10. Kwekkeboom KL, Hau H, Wanta B, Bumpus M. Patients' perceptions of the effectiveness of guided imagery and progressive muscle relaxation interventions used for cancer pain. Complement Ther Clin Pract. 2008;14(3):185-94.

11. Haro LM, Mondéjar R, Muñoz MM, Molina MJ, Olaverri A, Santiago JA. Tratamiento psicológico de las náuseas y vómitos anticipatorios inducidos por quimioterapia o radioterapia. Psicooncología. 2013;10(2-3):289-98.

12. Sleight AG, Duker LI. Toward a broader role for occupational therapy in supportive oncology care. Am J Occup Ther. 2016;70(4):7004360030p1-8.

13. Jensen W, Bialy L, Ketels G, Baumann FT, Bokemeyer C, Oechsle K. Physical exercise and therapy in terminally ill cancer patients: a retrospective feasibility analysis. Support Care Cancer. 2014;22(5):1261-8.

14. Cruzado JA. Tratamiento psicológico en pacientes con cáncer. España: Síntesis; 2010.

15. NORMA Oficial Mexicana NOM-012-SSA3-2012, Que establece los criterios para la ejecución de proyectos de investigación para la salud en seres humanos. Secretaría de Salud, 2012.

16. Chen SF, Wang HH, Yang HY, Chung UL. Effect of relaxation with guided imagery on the physical and psychological symptoms of breast cancer patients undergoing chemotherapy. Iran Red Crescent Med J. 2015;17(11):e31277.

17. Polat U, Arpaci A, Demir S, Erdal S, Yalcin S. Evaluation of quality of life and anxiety and depression levels in patients receiving chemotherapy for colorectal cancer: impact of patient education before treatment initiation. J Gastrointest Oncol. 2014;5(4):270-5.

18. Pandey M, Sarita GP, Devi N, Thomas BC, Hussain BM, Krishnan R Distress, anxiety, and depression in cancer patients undergoing chemotherapy. World J Surg Oncol. 2006;4:68.

19. De la Roca-Chiapas JM, Barbosa-Sabanero G, Martinez-Garcia JA, Martinez-Soto J, Ramos-Frausto VM, Gonzalez-Ramirez LP, et al. Impact of stress and levels of corticosterone on the development of breast cancer in rats. Psychol Res Behav Manag. 2016;9:1-6.

20. Segerstrom SC, Miller GE. Psychological stress and the human immune system: a meta-analytic study of 30 years of inquiry. Psychol Bull. 2004;130(4):601-30.

21. Masafia S, Rezaeib O, Ahadic H. Efficacy of biofeedback associated with relaxation in decreasing anxiety in women with breast cancer during chemotherapy. Procedia - Social and Behavioral Sciences. 2011;30:143-8.

22. Hayama Y, Inoue T. The effects of deep breathing on 'tension-anxiety' and fatigue in cancer patients undergoing adjuvant chemotherapy. Complement Ther Clin Pract. 2012;18(2):94-8.

23. Song $\mathrm{QH}, \mathrm{Xu} \mathrm{RM}$, Zhang QH, Ma M, Zhao XP. Relaxation training during chemotherapy for breast cancer improves mental health and lessens adverse events. Int J Clin Exp Med. 2013;6(10):979-84. 